\title{
ENVIRONMENTAL DEPENDENCE OF STAR FORMATION AND GALAXY TRANSFORMATION IN MERGING GALAXY CLUSTER ABELL 2255: AKARI'S POINT OF VIEW
}

\author{
HYUNJIN SHIM \\ Department of Earth Science Education, Kyungpook National University, Daegu 702-701, Korea \\ E-mail: hjshim@knu.ac.kr \\ (Received July 01, 2012; Accepted July 23, 2012)
}

\begin{abstract}
We investigate the role of galaxy environment in the evolution of individual galaxies through the AKARI observations of the merging galaxy cluster A2255. MIR diagnostics using $N 3-S 11$ colors are adopted to select star-forming galaxies and galaxies in transition between star-forming galaxies and quiescent galaxies. We do not find particular enhancement of star formation rates as a function of galaxy environment, reflected in cluster-centric distance and local surface density of galaxies. Instead, the locations of intermediate MIR-excess galaxies $(-1.2<N 3-S 11<0.2)$ show that star-forming galaxies are transformed into passive galaxies in the substructures of A2255, where the local surface density of galaxies is relatively high.
\end{abstract}

Key words: galaxies: clusters: individual (A2255); galaxies: photometry; infrared: galaxies

\section{INTRODUCTION}

Abell 2255 is a well-studied rich cluster at $z \sim 0.08$ which consists of a few hundreds member galaxies. From the X-ray observations showing elongated temperature gradient (Davis \& White, 1998), the cluster is considered to be at the stages of late merger. In general as a representative of high-density environments, galaxy clusters are thought to show less star formation than the field (e.g., Poggianti et al., 2009) due to the mechanisms that quench star formation including rampressure stripping, tidal forces, and galaxy harassment. However it is still under debate whether these mechanisms react differently when cluster-scale merging is incorporated (Hwang \& Lee, 2009): cluster-cluster merging can enhance or quench the star formation by driving external potential (Bekki, 1999), or by depriving gas (Fujita et al., 1999), respectively.

Mid-infrared (MIR) observations reveal how much star formation is actually hidden by dust (Bai et al., 2009) and work as good probe for young quiescent galaxies being sensitive of circumstellar dust emission around AGB stars (Ko et al., 2009). In this study, we investigate the role of local environment in the evolution of individual member galaxies in the cluster A2255.

\section{DATA}

The MIR photometry data over A2255 were obtained through CLEVL (CLusters of galaxies EVoLution studies) program, one of the AKARI mission programs (Im et al., 2008). We observed $\sim 1,200 \operatorname{arcmin}^{2}$ of A2255 using IRC02 Astronomical Observation Templates, with the filter composition of $[\mathrm{N} 3, \mathrm{~N} 4, \mathrm{~S} 7, \mathrm{~S} 11$, L15, and L24]. The on-source exposure time for each filter is roughly $\sim 140$ seconds.

The images are reduced using the IRC pipeline version 070104 (Y. Ita, T. Wada et al., 2007¹; provided in the form of an IRAF external package). After the reduction, the MIR fluxes in each filter are measured using SExtractor (Bertin \& Arnouts, 1996). The flux limit in each filter is $25,30,65,80,150$, and $400 \mu \mathrm{Jy}$

\footnotetext{
1 http://www.ir.isas.jaxa.jp/ASTRO-F/Observation/DataReduction/IRC/software/irc20070104.tgz.
} 


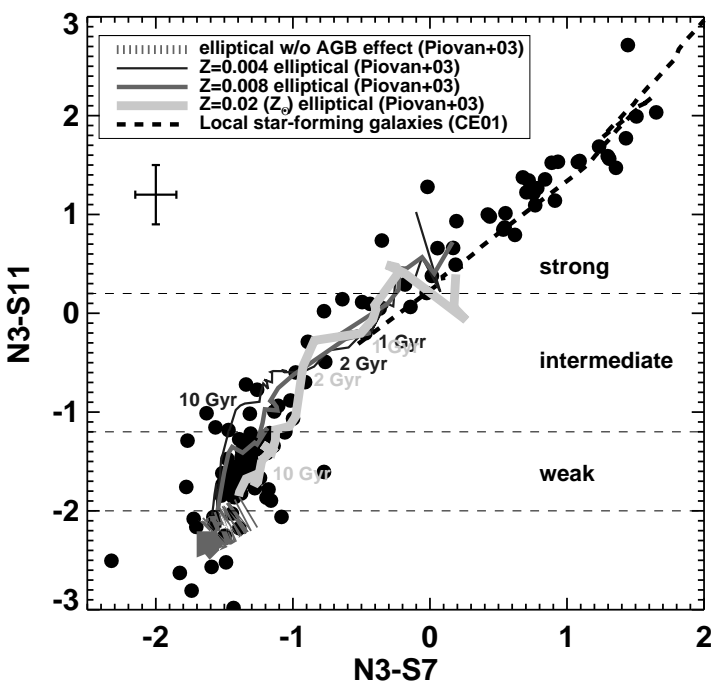

Fig. 1. $(N 3-S 11)$ vs. $(N 3-S 7)$ color-color plot of A2255 member galaxies. Overplotted are colors expected from local star-forming galaxies (dashed line; Chary \& Elbaz, 2001), elliptical galaxies with circumstellar dust emission around AGB stars (solid lines; Piovan et al., 2003), and elliptical galaxies without AGB dust emission considered (thick dotted line). The error bar indicates typical magnitude errors.

\section{at $3,4,7,11,15$, and $24 \mu \mathrm{m}$ respectively.}

In order to identify member galaxies in A2255, we used the spectroscopic redshifts from the SDSS Data Release 2 (SDSS DR2; Abazajian et al., 2004) and the BATC photometric redshifts from Yuan et al. (2003) The redshift cut for member galaxies is $0.068<z<$ 0.090 , and the median redshift is $\langle z\rangle=0.081$. Over the $\sim 1,200 \operatorname{arcmin}^{2}$, we identified 122 galaxies which are spectroscopically confirmed to be within the redshift cut. In addition to that, 170 galaxies with the corresponding photometric redshifts are identified, thus the total number of member galaxies used is 292 .

\section{MIR DIAGNOSTICS}

While the cluster member galaxies show a tight red sequence in their optical and near-infrared colormagnitude diagram which is defined by elliptical galaxies with similar age and/or metallicity, the sequence is not well-defined in the MIR color-magnitude diagram due to the large scatter in MIR colors reflecting stellar population age and dust emission (e.g., Ko et al., 2009). Figure 1 shows MIR color-color diagram for galaxies in
A2255.

Most galaxies have $N 3-S 11$ colors redder than -2.0 , expected from a pure stellar continuum of passive elliptical galaxies without any concern for dust emission from AGB stars (thick dotted line in Figure 1). These 'MIR-excess' galaxies can be divided into three classes according to the strength of the MIR color excess, reflected in $N 3-S 11$ colors: strong MIRexcess $(N 3-S 11>0.2)$, weak MIR-excess $(-2.0<$ $N 3-S 11<-1.2)$, and intermediate MIR-excess in between the two.

Strong MIR-excess galaxies have N3-S11 and N3$S 7$ colors consistent with those of local star-forming galaxies (dashed line; Chary \& Elbaz, 2001). Intermediate MIR-excess galaxies, $-1.2<N 3-S 11<0.2$, are considered to be passive galaxies with relatively young stellar population (less than few Gyrs) and/or recent star formation activity. In this work, we used these MIR diagnostics to study the role of cluster environment to star formation and/or evolution of member galaxies.

\section{ENVIRONMENTAL DEPENDENCE}

A2255 has a number of substructures that can affect the evolution of individual member galaxies in addition to the asymmetric velocity distribution reflecting the complicated kinematics within the cluster. As gauge of the local environment, we used the cluster-centric distance and the local surface density of galaxies. The local surface density of galaxies is expressed as $\Sigma_{5 t h}$, the density of galaxies (with either spectroscopic or photometric redshifts) within a circle whose radius is the distance to the 5th-nearest galaxy in comoving scale. If a galaxy cluster is dynamically relaxed, the local surface density of galaxies would monotonically decrease as the clustercentric distance increases: however, there exists several $\Sigma_{5 t h}$ excess peak compared to the simple 2D-projected NFW profile thus we defined these as substructures of A2255.

In Figures 2 a, b, and c, we showed the average specific star formation rates (SFRs) distribution as a function of local environment. The star formation rate is calculated from the total IR luminosity derived through SED fitting. There is no strong trend of increase or decrease of specific SFR with the cluster-centric distance, except that the specific SFR of galaxies at the outermost of A2255 increases. This specific SFR at the outermost bin reflects newly infalling galaxies into the 

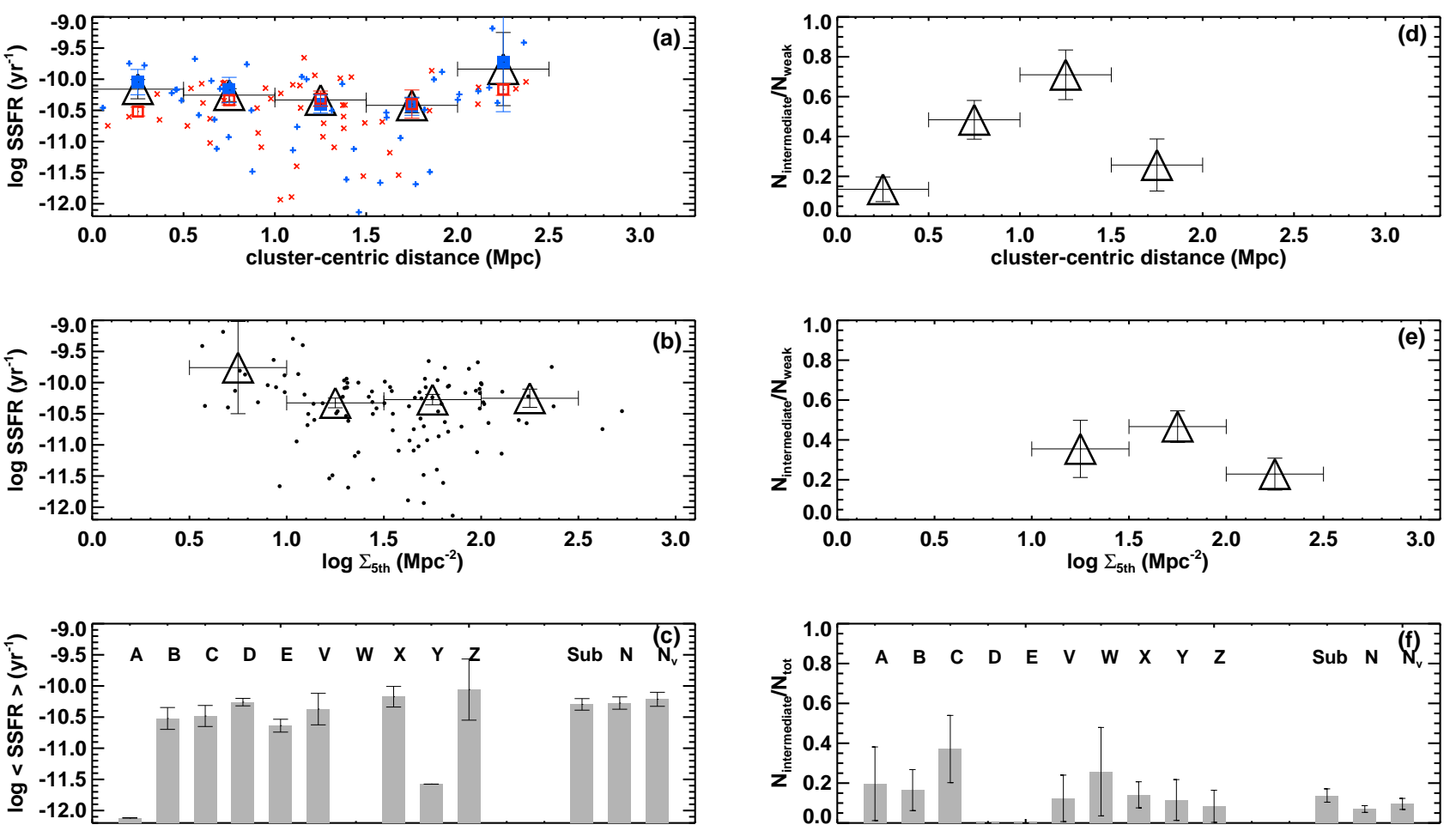

Fig. 2. Environmental dependence of the specific star formation rate (a, b, and $c$ ) and the fraction of intermediate MIR-excess galaxies (d, e, and f). (a) Crosses indicate specific star formation rates (SSFR) of individual galaxies, blue from galaxies at $z<0.08$ (blue velocity peak) and red from galaxies at $z>0.08$ (red velocity peak). Triangles with error bars indicate average SSFR in each cluster-centric distance bin. Blue filled squares and red open squares are average SSFR in each cluster-centric distance bin, calculated using galaxies at $z<0.08$ and $z>0.08$ respectively. (b) Dots are SSFR of individual galaxies, triangles average SSFR in each local galaxy surface density bin. (c) Average SSFR of galaxies in substructures A-Z of A2255. Additionally compared are 'Sub' (indicating average SSFR of galaxies in all substructures) and ' $\mathrm{N}_{v}$ ' (indicating average SSFR of galaxies with velocities at extreme blue/red end of velocity distribution $(z<0.075$ or $z>0.085)$ ), in addition to the result from all ('N') galaxies. (d) Ratio between the number of intermediate MIR-excess galaxies to number of weak MIR-excess galaxies as a function of cluster centric distance. (e) Same as (d), but as a function of local surface density of galaxies. (f) Fraction of intermediate MIR-excess galaxies, i.e., number of intermediate MIR-excess galaxies divided by number of total galaxies measured for substructures. Error bars in all panels are derived through bootstrapping.

cluster. If we divide galaxies using velocities (blue and red), galaxies at the blue velocity peak tend to have higher specific SFR than galaxies at the red velocity peak especially near the central region of the cluster. While specific SFR does not show a significant correlation with local galaxy surface density where a galaxy resides, it is more likely that galaxy kinematics are related to the amount of specific SFR at least in the region close to the cluster center.

On the other hand, the increase of the ratio between the number of intermediate MIR-excess galaxies and the number of weak MIR-excess galaxies as a function of cluster-centric distance is clearer (Figure $2(\mathrm{~d})$ ). The fraction of intermediate MIR-excess galaxies gets slightly higher in some substructures (C, W) still despite large scatter. Therefore the transition from starforming galaxies to passive galaxies seems to occur in the substructures with high local galaxy density. 

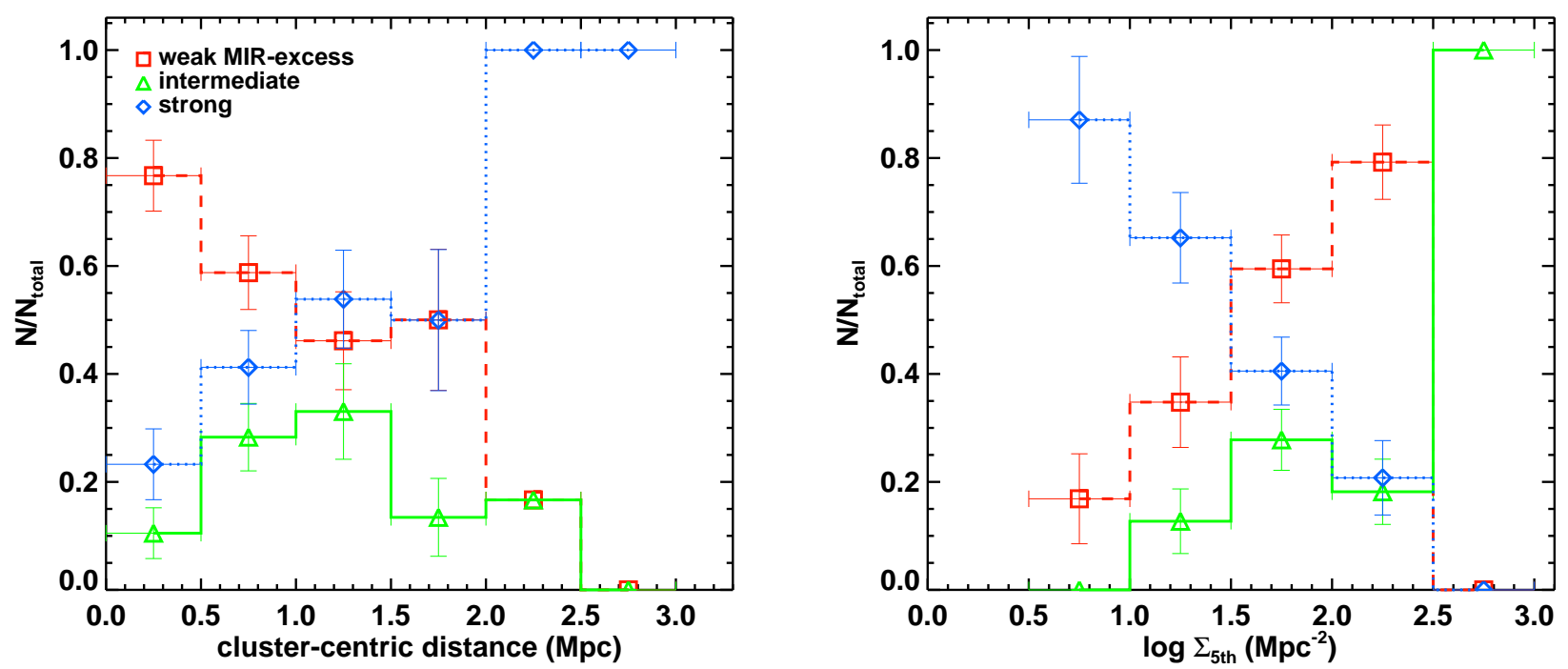

Fig. 3. Fractions of strong, intermediate and weak MIR-excess galaxies among the total galaxies as a function of cluster-centric distance (left) and local surface density of galaxies (right). The error bars are derived through bootstrapping.

\section{CONCLUSION}

It is difficult to tell whether star formation is enhanced or quenched due to the local environment within the merging galaxy cluster A2255. The spatial distribution of strong, intermediate and weak MIR-excess galaxies show a general color-density relation (Figure 3), confirming the increase in the fraction of strong MIRexcess galaxies in the low density region, outer part of the cluster and the fraction of weak MIR-excess galaxies on the other way around. It is worthwhile to note that the fraction of intermediate MIR-excess galaxies increases in the moderate local surface density region, which suggest that the transition from star-forming galaxies to passive galaxies occurs mainly in the substructures of A2255.

\section{REFERENCES}

Abazajian, K., et al., 2004, The Second Data Release of the Sloan Digital Sky Survey, AJ, 128, 502

Bai, L., et al., 2009, The Infrared Luminosity Functions of Rich Clusters, ApJ, 693, 1840

Bekki, K., 1999, Group-Cluster Merging and the Formation of Starburst Galaxies, ApJ, 510, L15

Bertin, E. \& Arnouts, S., 1996, SExtractor: Software for Source Extraction, A\&AS, 117, 393

Chary, R. \& Elbaz, D., 2001, Interpreting the Cosmic Infrared Background: Constraints on the Evolution of the Dust-enshrouded Star Formation Rate, ApJ, 556, 562
Davis, D. S. \& White, R. E. III, 1998, An X-Ray Temperature Map of the Merging Cluster Abell 2255, ApJ, 492, 57

Fujita, Y., et al., 1999, Is Galactic Star-Formation Activity Increased during Cluster Mergers?, PASJ, 51, L1

Hwang, H. S. \& Lee, M. G., 2009, Galaxy Activity in Merging Binary Galaxy Clusters, MNRAS, 2009, 397,2111

Im, M. et al., 2008, Evolution of Galaxy Clusters from an AKARI Mission Program, CLEVL, 2008, ASPC, 399, 382

Ko, J. et al., 2009, The Mid-infrared View of Red Sequence in Abell 2218 with AKARI, ApJ, 695, L198

Piovan, L., Tantalog, R., \& Chiosi, C., 2003, Shells of Dust around AGB Stars: Effects on the Intergrated Spectrum of Single Stellar Populations, A\&A, 408, 559

Poggianti, B. M., et al., 2009, The Evolution of Spiral, S0, and Elliptical Galaxies in Clusters, ApJ, 697, L137

Yuan, Q., Zhou, X., \& Jiang, Z., 2003, Multicolor Photometry of the Galaxies in A2255 by the Beijing-Arizona-Taiwan-Conneticut Survey and Sloan Digital Sky Survey, ApJS, 149, 53 